\title{
Local interaction in retinal ganglion cell mosaics can generate a consistent spatial periodicity in cortical functional maps
}

Jaeson Jang ${ }^{*}$, Se-Bum Paik

From 24th Annual Computational Neuroscience Meeting: CNS*2015

Prague, Czech Republic. 18-23 July 2015

Orientation map is one of the most studied functional maps in visual cortex, but the developmental mechanism of its consistent spatial periodicity is still elusive. Recently, a theoretical model suggested that a moiré interference pattern between ON and OFF retinal ganglion cell (RGC) mosaics can develop a quasi-periodic orientation map, but it is remained unclear how this can explain the constant periodicity of the maps [1]. Here we suggest a developmental model that a simple local interaction in RGCs can generate a consistent spatial periodicity of orientation preference, by inducing (i) a hexagonal lattice structure in ON/OFF RGC mosaics and (ii) a constant alignment angle between them.

First, we introduced a developmental model of a monotypic RGC mosaic to show that a local repulsive interaction can generate a hexagonal structure (Figure 1A).

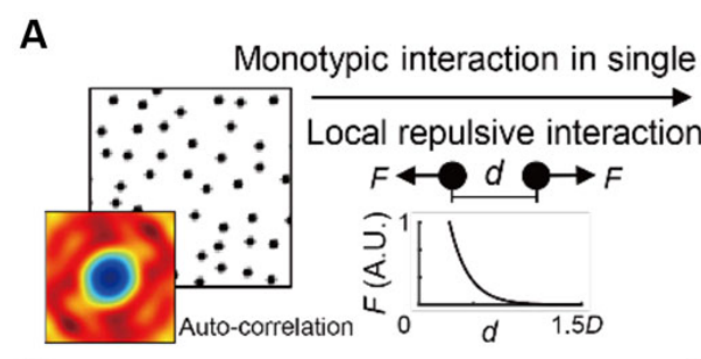

C

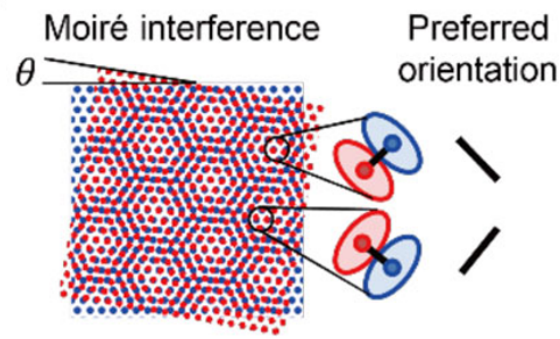

B

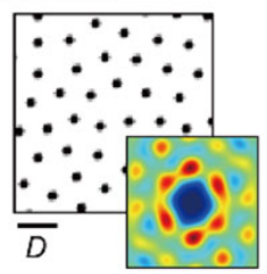

D

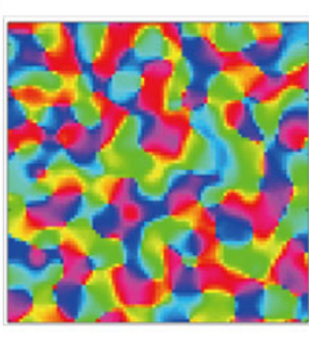

Heterotypic interaction between mosaics

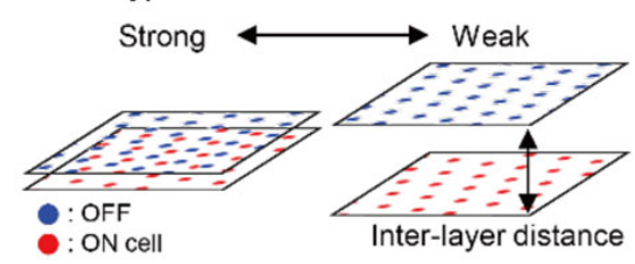

E

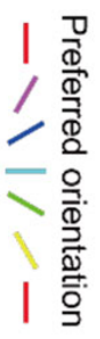

0.015

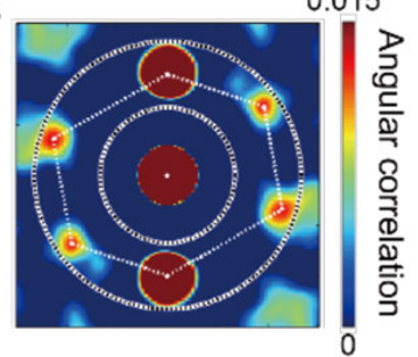

Figure 1 Simulation of RGC mosaics and orientation map. A. Development of monotypic mosaic and auto-correlation. B. Heterotypic interaction depends on inter-layer distance. C. Moiré interference pattern of ON/OFF RGC mosaics. D. Simulated orentation map. E. Hexagonal pattern in auto-correlation of orientation map.

\footnotetext{
* Correspondence: jaesonjang@kaist.ac.kr

Department of Bio and Brain Engineering, KAIST, Daejeon, 305-701, Republic of Korea
} original work is properly cited. The Creative Commons Public Domain Dedication waiver (http://creativecommons.org/publicdomain/ zero/1.0/) applies to the data made available in this article, unless otherwise stated. 
Previously, in the model study of the pairwise interaction point process, it was suggested that a local interaction alone cannot develop a long-range order in the mosaic structure [2]. We assumed a different type of local repulsive interaction that the cell positions can be gradually shifted by a repulsion from the neighbor cells and confirmed that this model can develop a long-range ordered structure that is well fitted to a hexagonal lattice.

Next, we assumed that there also exists a heterotypic repulsive interaction between ON and OFF RGC mosaics and examined how this can affect the alignment between the two mosaics (Figure 1B). When the inter-layer distance between ON/OFF mosaics was varied within a proper interval, the hexagonal structure was preserved in each mosaic, but the alignment angle $(\theta)$ between the two mosaics was restricted within a certain range of angles, and this induced a constant spatial periodicity in the $\mathrm{ON} /$ OFF interference pattern (Figure 1C). As observed in the moiré interference, we confirmed a consistent hexagonal periodicity in the cortical orientation map that are simulated by statistical wiring model from the developed RGC mosaics (Figure 1D,E) [3].

\section{Conclusions}

Our result suggests that a local repulsive interaction in RGC mosaics can generate a hexagonal structure in ON/ OFF RGC mosaics and a restricted alignment between them. The interference between mosaics induces a consistent spatial periodicity in cortical orientation map as predicted by the moiré interference pattern.

Published: 18 December 2015

\section{References}

1. Paik SB, Ringach DL: Retinal origin of orientation maps in visual cortex. Nat Neurosci 2011, 14:919-925.

2. Hore VR a, Troy JB, Eglen SJ: Parasol cell mosaics are unlikely to drive the formation of structured orientation maps in primary visual cortex. Vis Neurosci 2012, 29(6):283-299.

3. Ringach DL: Haphazard wiring of simple receptive fields and orientation columns in visual cortex. J Neurophysiol 2004, 92(1):468-476.

doi:10.1186/1471-2202-16-S1-P192

Cite this article as: Jang and Paik: Local interaction in retinal ganglion cell mosaics can generate a consistent spatial periodicity in cortical functional maps. BMC Neuroscience 2015 16(Suppl 1):P192.

\section{Submit your next manuscript to BioMed Central} and take full advantage of:

- Convenient online submission

- Thorough peer review

- No space constraints or color figure charges

- Immediate publication on acceptance

- Inclusion in PubMed, CAS, Scopus and Google Scholar

- Research which is freely available for redistribution

Submit your manuscript at www.biomedcentral.com/submit 